\title{
A comparative study of the efficiency of chart versus computer-generated contrast sensitivity testing in glaucoma patients and controls.
}

\author{
BY SALLY CHETRIT, OD; MELISSA GAUDET, OD; WALTER WITTICH, MA; \\ IAN L. BAILEY, OD, MS, FCOPTOM, FAAO; OLGA OVERBURY, PHD
}

A $\mathrm{s}$ an integral part of a patient's primary care team, the optometrist plays a key role in the diagnosis and co-management of glaucoma. In Canada alone, one in 100 individuals over the age of 40 will develop glaucoma, a leading cause of blindness. ${ }^{1}$ Vision loss caused by this ocular disease is preventable, thus emphasizing the importance of early diagnosis and treatment.

Glaucoma is a condition which, in most cases, is accompanied by an elevation of the intra-ocular pressure (IOP). This increase in IOP is

ABRÉGÉ

Objectif : Le but de la présente étude était d'évaluer l'efficacité de tests de sensibilité au contraste imprimés ou générés par ordinateur chez des patients atteints de glaucome ou ayant une bonne vision. Méthode: Nous avons mesuré la sensibilité au contraste d'un groupe de 64 individus composé de 30 jeunes et 18 aînés (groupe contrôle) ainsi que 16 patients atteints de glaucome. Dans un premier temps, nous avons utilisé deux tests pour déterminer la sensibilité au contraste (SC). Le premier est l'échelle de MARS et le second, un test de Bailey informatisé comprenant une tâche de recherche de nombres. La deuxième étape consistait à déterminer la sensibilité au contraste spatial (SCS) pour des cibles de différentes fréquences spatiales. Pour ce faire, nous avons utilisé l'échelle Vistech ainsi qu'une tâche informatisée mise au point par Faubert. Résultats: Les résultats obtenus aux tests de SC démontrent un déclin chez les patients atteints de glaucome comparativement aux deux groupes contrôles $(p<0.001)$. Le test mesurant le
SCS démontre un déclin de sensibilité chez les participants ainés $(p<0.001)$ ainsi que chez les patients atteints de glaucome $(p<0.001)$. Conclusion. Les données obtenues indiquent que le SCS semble supérieur pour différencier les trois groupes étudiés. Le test de Faubert ainsi que le Vistech mesurant la sensibilité au contraste à diverses fréquences spatiales démontrent un déclin statistiquement significatif dans la mesure de SC, particulièrement dans le spectre médian des fréquences spatiales. Comme ces différences sont importantes, elles deviennent plus faciles à détecter même lorsque le matériel informatique n'est pas calibré. De plus, ces tâches sont plus facilement exécutées. Ces résultats soulignent l'importance des différentes fréquences spatiales dans la détection et le dépistage du glaucome. D'autre part, le test informatique n'apporte pas plus de bénéfices que la version imprimée.

Mots clés : glaucome, sensibilité au contraste, sensibilité au contraste spatial, âge, procédures de test a consequence of increased resistance in the eye's trabecular meshwork which, as a result, slows down the filtration of the aqueous humor. The elevated IOP causes mechanical damage and a decrease in blood perfusion at the level of retinal ganglion cells, and there is progressive optic nerve atrophy and visual damage. ${ }^{2}$ The disease mechanism, however, is different for normal tension glaucoma.

While glaucoma may affect many visual functions, the ones of interest in this study were contrast sensitivity and spatial contrast sensitivity. Both these aspects of spatial vision can be very strong predictors of everyday performance and may provide insight into a patient's quality of life. Contrast sensitivity (CS) is defined as the measure of an individual's ability to detect a difference in luminance between two distinctly defined areas, whereas spatial contrast sensitivity (SCS) also considers the size of a target's spatial components ${ }^{3}$, for example by using sinusoidal gratings with different spatial frequencies as the test targets.

The standard clinical evaluation of CS and SCS is executed by means of printed test charts. Even though contrast and spatial configurations are controlled very precisely, these tests require an external light source, which often makes it difficult to 


\section{ABSTRACT}

ABSTRACT: Purpose. The goal of this study was to assess the efficiency of chart vs. computergenerated contrast sensitivity tests in glaucoma patients and controls. Methods. A total of 64 individuals (30 young controls, 18 older controls, 16 glaucoma patients) were tested for contrast sensitivity using 4 different tests. Two tests determined contrast sensitivity (CS) for detecting large targets with sharp borders. One of these was the MARS printed chart, and the other a computerized number search test by Bailey. The second assessment determined spatial contrast sensitivity (SCS) for sinusoidal grating targets at several spatial frequencies. One of these was the printed Vistech chart, the other a computerized test by Faubert. Results. Both CS tests showed a decrease in the glaucoma group versus both the control groups $(p<0.001)$. The tests for SCS demonstrated a decrease in sensitivity both with age $(p<$ 0.001 ) and in the presence of glaucoma ( $p<$ 0.001 ) across all spatial frequencies. Conclusion. The data indicated that SCS was superior in separating the three study groups. Neither of the computer-generated tests was more sensitive than its printed counterpart.

Key Words: glaucoma, contrast sensitivity, spatial contrast sensitivity, age, testing procedures.

achieve adequate and uniform illumination across the chart. This problem can be overcome with the use of computer monitors to display CS and SCS stimuli. The quality of computer displays has improved in the last decade, making the standard PC capable of generating high-resolution images suitable for presenting test stimuli. ${ }^{4}$

The objective of the present study was to compare the efficiency of chart vs. computer-generated CS and SCS tests. Specifically, it was hypothesized that the computer-generated tests would prove to be more sensitive to visual defects caused by glaucoma.

\section{Methods}

Both the Health Sciences Research Ethics Committee at Université de Montréal and the Sir Mortimer B. Davis Jewish General Hospital Institutional Review Board approved the study protocol as adhering to the Tenets of the Declaration of Helsinki for research conducted with humans.

\section{Participants}

Sixty-four individuals participated in this study and were divided into 3 categories: Group 1 consisted of 30 young controls (mean age $=23$ years, range 21-28), Group 2 consisted of 18 older controls (mean age $=60$ years, range 50-80) and Group 3 consisted of 16 primary open-angle glaucoma patients (mean age $=71$ years, range 51-85). Participants from Group 1 with normal or corrected-to-normal vision were recruited primarily from the student body of the Université de Montréal, École d'Optométrie. Group 2, recruited from the Clinique Universitaire de la $V i s i o n$, consisted of patients with visual acuity better than $20 / 40(6 / 12)$ and no identified ocular disorders. Group 3 was recruited from the patient population of the Ophthalmology Department at the SMBD Jewish General Hospital. These patients had received a diagnosis of primary open-angle glaucoma by a glaucoma specialist. For the purpose of the present study, a patient diagnosed with glaucoma was defined as one having an IOP greater than $21 \mathrm{mmHg}$ on at least two occasions, as well as a diagnostic visual field defect or pathological cup-todisc ratio. Informed written consent was obtained from each subject before testing.

\section{Materials \& Procedure}

The evaluation of CS and SCS was accomplished by using four different tests: the Faubert test (Montréal, Québec), the Vistech chart (Dayton, Ohio), the MARS chart (Chappaqua, NY), as well as the Bailey test (Berkeley, California). Every testing session began with an evaluation of the visual acuity using the ETDRS visual acuity chart (Lighthouse, NY), placed at a distance of 4 meters. All participants had visual acuity of $20 / 40$ or better. For each participant, only one eye was tested. For Groups 1 and 2, the eye to be tested was chosen randomly. For Group 3 , the tested eye was one diagnosed with glaucoma.

Evaluation of CS, using each of the four tests previously mentioned, followed visual acuity testing. The test order was randomized for each participant to avoid order effects from fatigue, variations in concentration and practice. For each test, the measurements were taken monocularly with the observer wearing the appropriate refractive correction.

The Faubert test is a computergenerated SCS test composed of sinusoidal gratings, varying in both spatial frequency $(1.5,3,6,12$, and $18 \mathrm{cpd}$ ) and in contrast, whereby increments were adjusted using the 
QUEST procedure. ${ }^{5}$ The observer's eye was 50 centimeters from a calibrated computer screen (mean luminance $\left.55 \mathrm{~cd} / \mathrm{m}^{2}\right)$. Each test target was presented for 750 milliseconds, after which the observers were asked to indicate the direction in which the gratings were oriented: vertically or horizontally. The observers were required to respond to each trial and they were obliged to guess when they were uncertain, even if the screen was perceived as uniformly grey (2-alternative forcedchoice paradigm).

The Vistech chart, used to evaluate SCS, is composed of circular sine wave gratings, placed in five rows and nine columns. The five rows vary in spatial frequency (1.5, $3,6,12$, and $18 \mathrm{cpd}$ ), while the $9 \mathrm{col}-$ umns vary in contrast. This test was performed at a distance of 3 meters with the chart uniformly illuminated by an external light source (mean luminance $55 \mathrm{~cd} / \mathrm{m}^{2}$ ). Observers were instructed to begin with the top row, identifying the last patch in which gratings could be discerned and then determining the direction in which the gratings were tilted: left, right or up. Even if no direction could be perceived, the participant was asked to guess (3-alternative forced-choice paradigm). This procedure is known to provide improved accuracy. ${ }^{6}$

The MARS chart is a CS test consisting of 48 letters of equal size arranged in eight rows of six letters each. Contrast varies from $91 \%$ (-0.04 log units) to $1.2 \%(-1.92 \log$ units) with the contrast of each letter decreasing by a factor of 0.04 $\log$ units. The chart was placed on a reading stand 50 centimeters from the patient (mean luminance $88 \mathrm{~cd}$ /

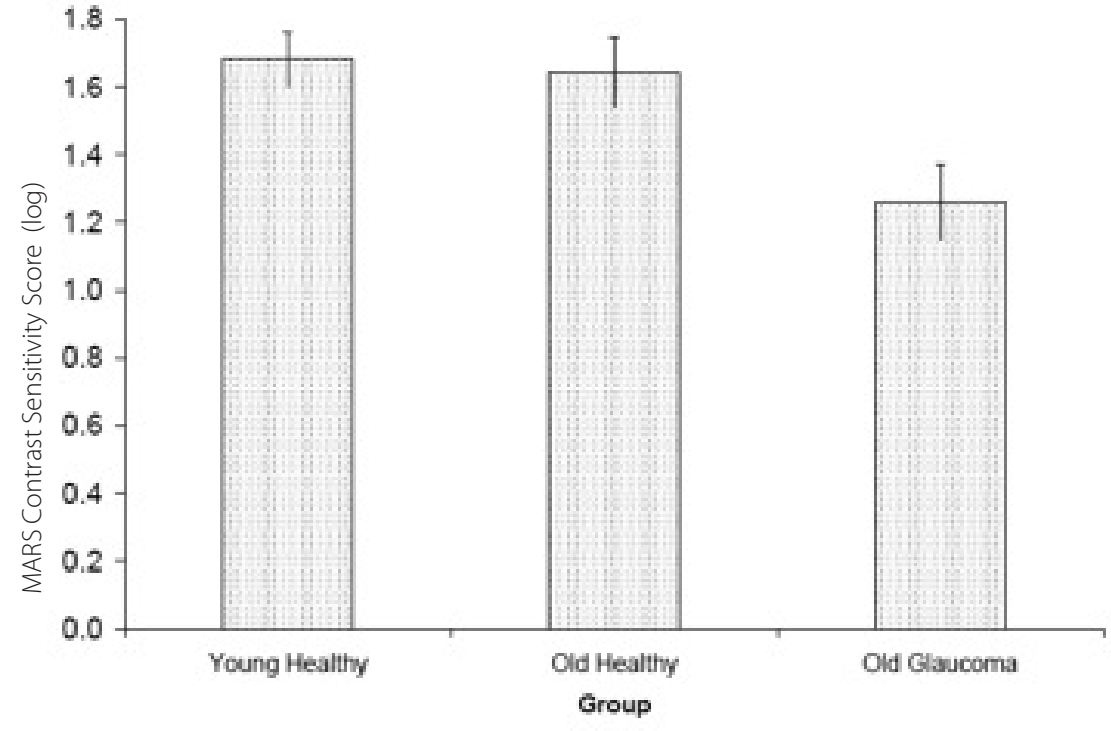

Figure 1: Mean contrast sensitivity scores on the MARS chart across three subject groups. Error bars represent $95 \%$ confidence intervals. Glaucoma patients had statistically significantly lower scores compared to both control groups.

$\mathrm{m}^{2}$, spatial frequency of $1.83 \mathrm{cpd}$ ). Subjects were instructed to read all the letters on the chart, beginning with the highest contrast letter. Testing ended when two consecutive letters were missed. ${ }^{7}$

The Bailey test is a computer-generated CS test that is in a prototype stage but is routinely used within the optometry clinics of the University of California at Berkeley for both clinical and research purposes. This is a test in which targets are the numbers 1 through 8 distributed in randomly assigned locations over the screen. These large $(40 \mathrm{~mm})$ numbers vary systematically in the degree of contrast, with 1 being at maximum contrast and 8 being at the minimum with a 0.3 log-unit difference from one contrast to the next. The test is designed with a standard sequence of 6 display screens, each presented for 20 seconds, so that there are two presentations each at 24 contrast levels ( 0.0 to $2.3 \mathrm{log}$ units). The stimuli were displayed on a calibrated computer screen at a viewing distance of 50 centimeters (spatial frequency of $1.83 \mathrm{cpd}$ ). The task for the patient was to locate the numbers in sequence. (The Bailey test was added to the protocol in order to provide a computer-generated way of testing CS; however, due to methodological problems, the calibration of the actual contrast levels was not accurate. As a consequence the numerical values do not indicate the actual contrasts, but they do, nevertheless, reliably reflect the order of the contrast levels. The resulting shift in CS results will be addressed in the discussion section in more detail.) 


\section{Results}

A partial Latin-square was used in the randomization of the testing sequence. The data collected from the MARS and Bailey tests were analyzed using a one-way analysis of variance (ANOVA). Figures 1 and 2 display the mean CS scores for both the MARS and the Bailey tests for all groups. Using the MARS test, a statistically significant main effect of group was detected, $F(2,61)=20.83$, $p<.001, \eta^{2}=.41$, whereby mean CS scores did not differ between the two groups of healthy younger and older observers; however, both groups differed significantly from the glaucoma group, $p<.001$, respectively (Tukey correction). Using the Bailey test, the same main effect was detected, $F(2,61)=22.06, p<$ $.001, \eta^{2}=.42$. Again, both younger and older normals could not be distinguished but both groups differed from the glaucoma participants, $p$ $<.001$, respectively (Tukey correction).

A two-way factorial ANOVA (5 spatial frequencies $\mathrm{x} 3$ test groups) was used for both the Vistech and the Faubert test in order to consider a second variable, spatial frequency. Mean values are displayed in Figures 3 and 4 . Note that the units for the Vistech analysis were not transformed into $\log$ units. The conversion was not possible since some of the glaucoma patients scored 0 on some of the spatial frequencies (they were unable to detect any gratings at the lower frequencies). For the Faubert test, the analysis revealed a statistically significant interaction effect, $F(8,244)=4.28, p<.001, \eta^{2}=$ .12 , whereby all three groups dem-

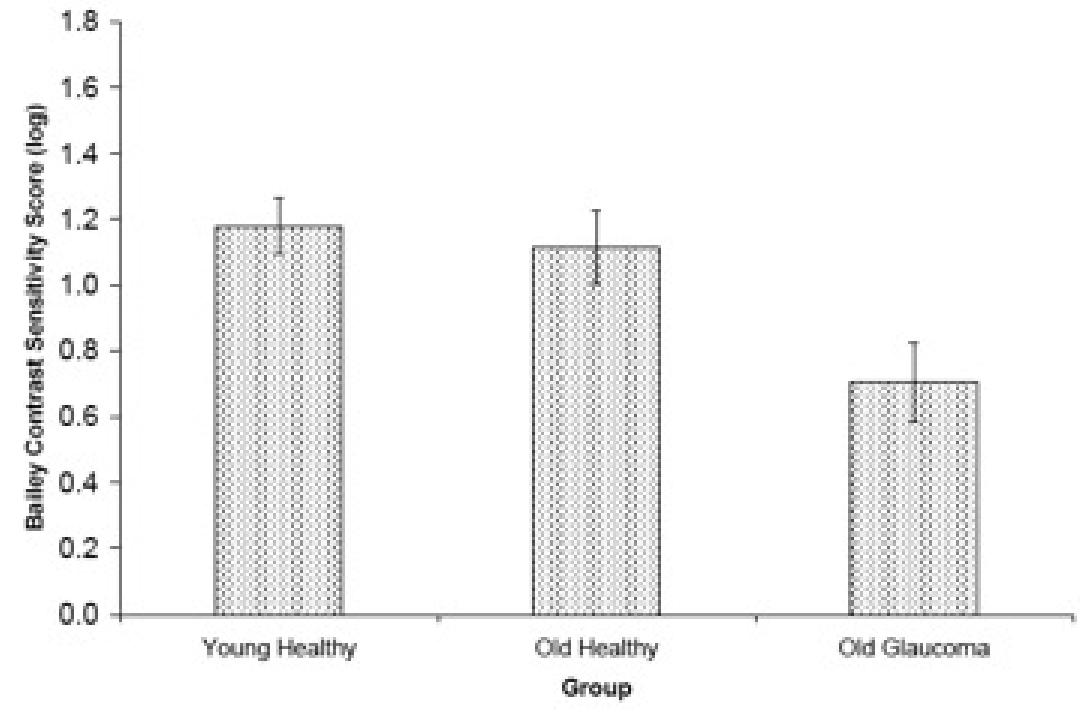

Figure 2: Mean contrast sensitivity scores on the Bailey test across three subject groups. Error bars represent 95\% confidence intervals. Glaucoma patients had statistically significantly lower scores compared to both control groups.

onstrated a decrease in CS as spatial frequency increased. Given that normal observers showed better $\mathrm{CS}$, this decrease was steeper when compared to the change in the glaucoma group. For the Vistech test, the analysis revealed an equivalent interaction effect, $\mathrm{F}(8,244)=4.80$, $\mathrm{p}<.001, \eta^{2}=.14$, with a similar pattern of change in the group scores across spatial frequencies.

\section{Discussion}

The present results indicated that the MARS and the Bailey tests, measuring CS, did not find any significant difference between the younger and older control groups. The failure to detect an age effect may be explained by the fact that both the MARS and Bailey tests assess the processing of only low spatial frequencies ${ }^{8}$, which are known to remain unaffected by age. $^{9-12}$ In comparison, the Faubert and Vistech tests, measuring contrast sensitivity over a range of spatial frequencies, demonstrated a statistically significant decrease in CS, notably in the middle spatial-frequency range. Since these differences were considerably larger, they become easier to detect, even without calibrated computer displays, and are more easily assessed, thereby emphasizing the importance of these frequencies in the detection of glaucoma. This decline may result from a combination of age-related optical and neural changes. Optical changes, such as age-related miosis, increased lenticular light scatter and ocular aberrations, may lead to reduced retinal illuminance and reduced contrast in the optical image that decrease CS, particularly at high spatial frequencies. ${ }^{12}$ Neural changes with aging are

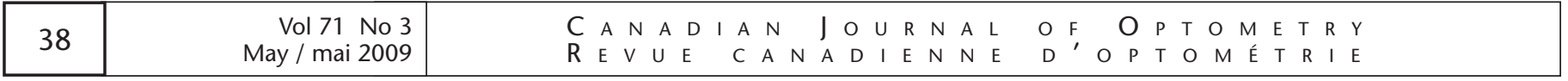




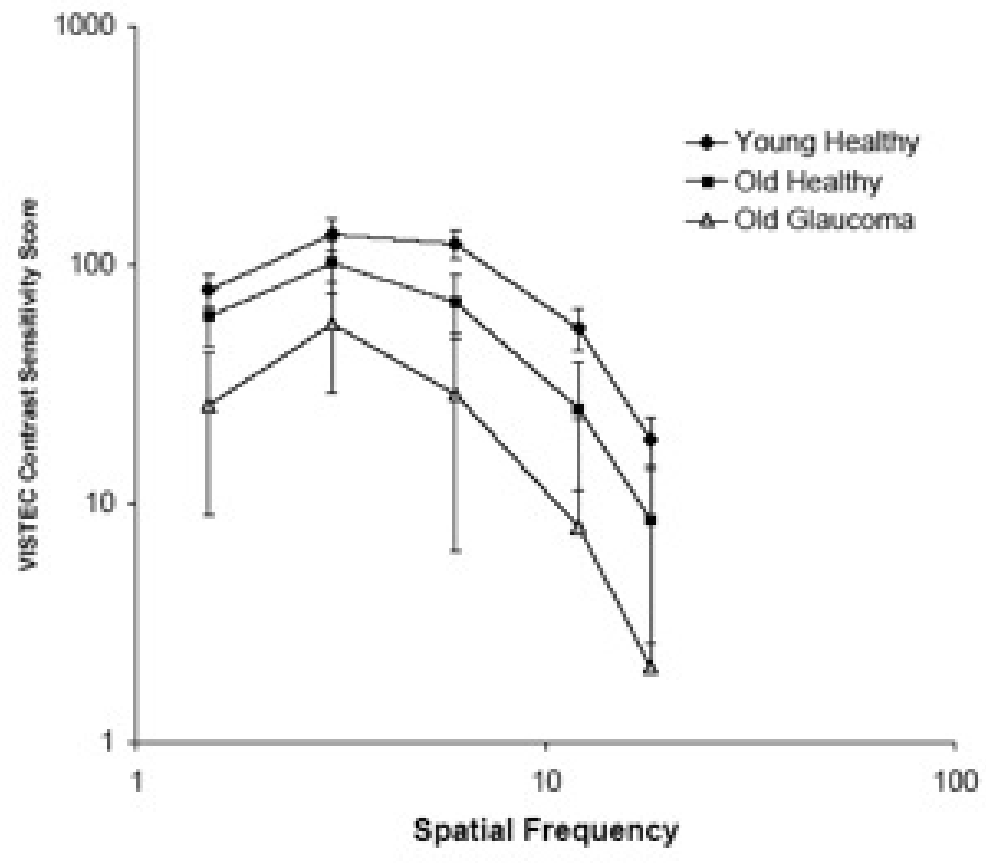

Figure 3: Mean contrast sensitivity scores across 5 spatial frequencies on the Vistech chart for three subject groups. Error bars represent 95\% confidence intervals. Scores differed among all three groups across all spatial frequencies.

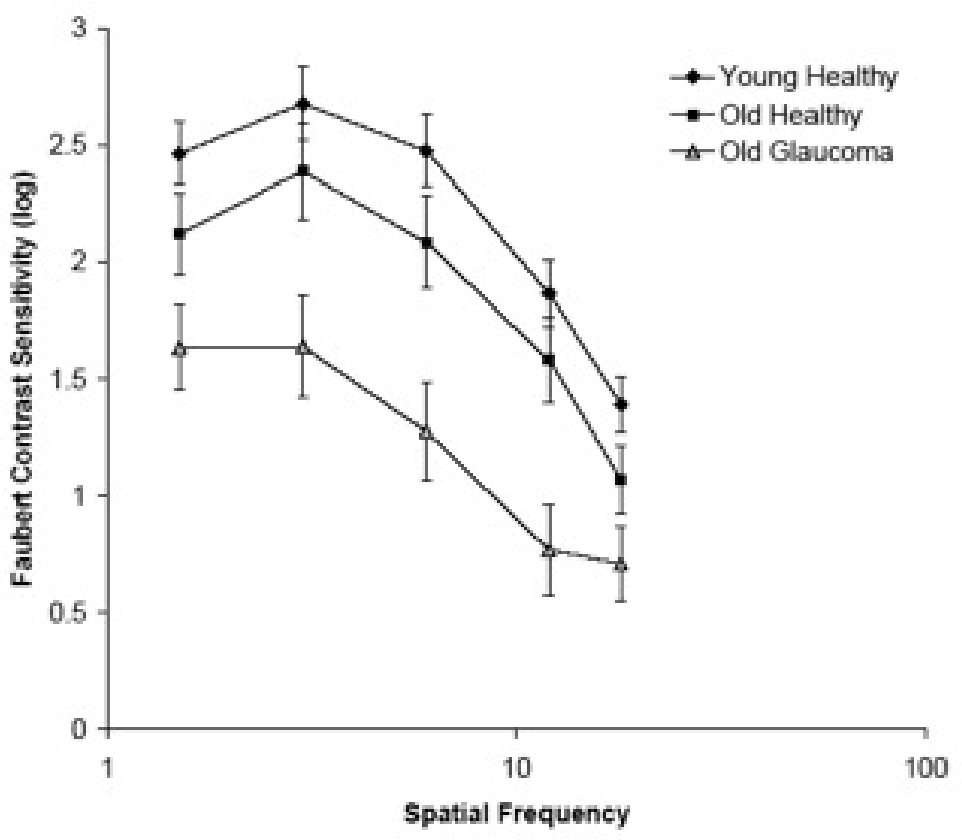

Figure 4: Mean contrast sensitivity scores across 5 spatial frequencies on the Faubert test for three subject groups. Error bars represent 95\% confidence intervals. Scores differed among all three groups across all spatial frequencies. attributed to neuronal loss occurring at various levels along the visual pathway between the retina and the cortex. It has been shown that these neural changes are responsible for the reduced CS at middle spatial frequencies. ${ }^{13}$

When assessing the effect of glaucomatous damage on CS, all four tests demonstrated a statistically significant loss of CS in the glaucoma group compared to the healthy control groups, across the range of spatial frequencies. This is expected from the diffuse ganglion cell damage occurring in glaucoma patients. Previous studies have found evidence of damage to both the magnocellular and parvocellular pathways in glaucoma, resulting in loss of visual information. ${ }^{14} \mathrm{~A}$ decrease of CS in the lower spatial frequencies has been attributed to magnocellular ganglion cell damage, while a decline in high spatial frequency CS has been associated with parvocellular ganglionic cell damage ${ }^{14}$.

When comparing the efficiency of printed chart tests to that of computer-generated CS tests, there was no difference in the ability of detecting glaucomatous change. All four tests were able to distinguish between the CS values for the healthy and the glaucomatous groups. Closer examination of the CS scores on the Bailey test (see Figure 2) as compared to the MARS test (see Figure 1) shows the CS values were systematically lower across all participants on the computer-generated task. This discrepancy suggested a significant difference in the calibration of the contrast levels; however, this problem was detected too late to be addressed during the study period. 
Therefore, the absolute contrast values on the Bailey test should be treated as suspect. Still, we believe that a systematic relationship exists between the nominal and the real CS values on the Bailey test, indicating that the interpretation of the results with regard to separating the glaucoma group from normal observers still holds.

In order to minimize the effect of other common eye disorders such as cataracts, age-related macular degeneration and diabetic retinopathy, only eyes with best corrected visual acuities of $20 / 40(6 / 12)$ or better were included in this study. While it is possible to have cataracts, for example, and still have such acuities, our assumption was that the influence of cataracts is minimal in these cases.

CS tests provide a comprehensive assessment of visual function across a wide range of contrasts and spatial frequencies that accurately reflect vision in everyday settings. CS testing holds promise as a means to measure functional changes noninvasively in glaucoma patients who maintain adequate visual acuity.

While automated technology seems to be the new trend in the diagnosis of a wide variety of pathologies, at least in the case of contrast sensitivity, the computer-generated tests used in this study failed to deliver the superiority and precision expected. As a result, the use of printed contrast sensitivity charts may, with good reason, remain the method of choice in the evaluation of this aspect of visual functioning.

\section{Acknowledgements}

The authors thank the participants of this study for their time, Jocelyn Faubert, P.h.D for his assistance with the computer-generated tests used in this study and Oscar Kasner, M.D. for access to his patient files.

\section{References}

1. Canadian Ophthalmological Society. What is glaucoma?, 2009; v. 2009.

2. Rhee DJ. Glaucoma. New York: McGraw-Hill Medical Pub. Division, 2003; xv, 441.

3. Hawkins AS, Szlyk JP, Ardickas Z, et al. Comparison of contrast sensitivity, visual acuity, and Humphrey visual field testing in patients with glaucoma. J Glaucoma 2003;12(2):134-8.

4. Thomson Software Solutions. Overview. 2009; v. 2009.

5. Watson AB, Pelli DG. QUEST: A Bayesian adaptive psychometric method. Perception and Psychophysics 1983;33(2):113-20.

6. Vistech Consultants Inc. VCTS 6500 operator's manula, 1988.

7. Arditi A. Improving the design of the letter contrast sensitivity test. Invest Ophthalmol Vis Sci 2005;46(6):2225-9.

8. The Mars Perceptrix Corporation. Mars letter contrast sensitivity test: User manual, 2003.

9. Elliott DB. Contrast sensitivity decline with ageing: a neural or optical phenomenon? Ophthalmic Physiol Opt 1987;7(4):415-9.

10. Morrison JD, McGrath C. Assessment of the optical contributions to the agerelated deterioration in vision. Q J Exp Physiol 1985;70(2):249-69.

11. Elliott D, Whitaker D, MacVeigh D. Neural contribution to spatiotemporal contrast sensitivity decline in healthy ageing eyes. Vision Res 1990;30(4): 541-7.
12. Pardhan S. Contrast sensitivity loss with aging: sampling efficiency and equivalent noise at different spatial frequencies. J Opt Soc Am A Opt Image Sci Vis 2004;21(2):169-75.

13. Gao H, Hollyfield JG. Aging of the human retina. Differential loss of neurons and retinal pigment epithelial cells. Invest Ophthalmol Vis Sci 1992;33(1):1-17.

14. McKendrick AM, Badcock DR, Morgan WH. Psychophysical measurement of neural adaptation abnormalities in magnocellular and parvocellular pathways in glaucoma. Invest Ophthalmol Vis Sci 2004;45(6):1846-53 .

Sally Chetrit ${ }^{1}$ OD; Melissa Gaudet ${ }^{1}$ OD; Walter Wittich ${ }^{2,5} \mathrm{MA}$;

Ian L. Bailey ${ }^{3}$ OD, MS, FCOptom, FAAO; Olga Overbury ${ }^{1,4,5} \mathrm{PhD}$

1. Université de Montréal, École d'Optométrie, Montréal, Canada

2. Department of Neurology \& Neurosurgery, McGill University, Montréal, Canada

3. School of Optometry, University of California Berkeley, United States of America

4. Department of Ophthalmology, SMBD Jewish General Hospital, McGill University, Montréal, Canada

5. Centre de recherche interdisciplinaire en réadaptation du Montréal métropolitain (CRIR), Montreal, Canada 